\title{
Kinetic Studies of the Alternating Copolymerization of Butadiene with Acrylonitrile Catalyzed by the Ethylaluminum Dichloride - Vanadyl Chloride System
}

\author{
Junji Furukawa, Eiichi Kobayashi,* and Yutaka IsEda** \\ Department of Synthetic Chemistry, Kyoto University, Kyoto, Japan.
}

(Received September 1, 1969)

\begin{abstract}
The mechanism of the alternating copolymerization of butadiene with acrylonitrile catalyzed by $\mathrm{EtAlCl}_{2}$ and $\mathrm{VOCl}_{3}$ was investigated experimentally using various concentrations of catalysts at varying temperatures. A step-growth polymerization was postulated from the characteristic feature of polymerization that the molecular weight of a resultant polymer increases in the course of polymerization. A linear correlation was found to exist between the ratio of the yield to the degree of polymerization and the yield, which suggests that polymerization proceeds with rapid initiation and chain transfer reactions. The overall rate of polymerization was found to be expressed as a function of $\left(\mathrm{EtAlCl}_{2}\right)^{3 / 2} \times\left(\mathrm{VOCl}_{3}\right)^{1 / 2}$ and the concentration of the active species as a function of $\left(\mathrm{EtAlCl}_{2}\right)^{1 / 2} \times\left(\mathrm{VOCl}_{3}\right)^{1 / 2}$. These facts suggest that the active species is generated from the dissociation of the $\mathrm{EtAlCl}_{2}-\mathrm{VOCl}_{3}$ complex through a redox mechanism and the propagation reaction takes place between the polymer radical and a complexed monomer of butadiene-acrylonitrile-Et $\mathrm{AlCl}_{2}$. Vanadium compound acts not only as a generator of the active species but also as a useful cocatalyst capable of regenerating the complexing agent for the monomer and preventing the polymer from gel formation.

KEY WORDS Alternating Copolymerization / Butadiene / Acrylonitrile / Vanadyl Chloride-Ethylaluminum Dichloride / Rate / Molecular Weight / Mechanism /
\end{abstract}

The alternating copolymerization of acrylonitrile with olefins such as styrene and propylene was successfully achieved by Hirooka et al. by the use of ethylaluminum dichloride as a complexing agent for acrylonitrile. ${ }^{1,2}$ A similar copolymerization of butadiene with acrylonitrile was carried out independently by Gaylord et al. ${ }^{3}$ and by us. ${ }^{4-6}$ Gaylord et al. copolymerized butadiene with acrylonitrile complexed with alkyl aluminum halide or zinc halide in the presence of peroxide as radical initiator. They drew the conclusion that the copolymerization was an alternating one from the fact that the copolymer composition was always $1: 1$, but the degree of alternation had not been determined because the copolymer was

* Institute for Chemical Research, Kyoto University, Uji, Japan.

** Institute for Chemical Research, Kyoto University, Uji, Japan.

On leave from Department of Research and Development, Bridgestone Tire Co., Kodaira, Tokyo, Japan. a sparingly soluble powdery one. On the other hand, the preparation of a soluble elastic alternate copolymer was successfully carried out by us by the additional use of transition metal compounds such as vanadium, titanium $^{4-6}$ and chromium $^{7}$ compounds as cocatalysts for the copolymerization of butadiene with acrylonitrile complexed with alkylaluminum halide. The addition of the cocatalyst was found to effect regeneration of the aluminum complexing agent. Less than 5\% of the vanadium compound (with respect to the aluminum compound) was sufficient to increase the activity of the latter. ${ }^{4-6}$ With respect to the formation of the soluble copolymer and the alternating tendency, it was found that the addition of the vanadium compound was also effective for aluminum chloride or zinc chloride ${ }^{7}$ complexing agents, since the absence of the vanadium compound resulted in the formation of a sparingly soluble copolymer, which would not otherwise have occurred. Furthermore the alternating 
tendency estimated from the NMR analysis of the soluble part seemed to be rather poor compared with the $\mathrm{AlCl}_{3}-\mathrm{VOCl}_{3}$ system. This report deals with a kinetic study of the alternating copolymerization of butadiene with acrylonitrile using the $\mathrm{EtAlCl}_{2}-\mathrm{VOCl}_{3}$ catalyst system to elucidate the mechanism of copolymerization.

\section{EXPERIMENTAL}

Acrylonitrile (AN) was refluxed over $\mathrm{CaH}_{2}$ for about 4 hours, and subsequently distilled into the Molecular Sieves 3A before use. Butadiene (BD) of polymerization grade supplied by the Japan Synthetic Rubber Co. was purified by passing through columns packed with potassium hydroxide pellets, calcium chloride, activated alumina and the Molecular Sieves 3A, followed by filtering off frozen water after cooling to $-78^{\circ} \mathrm{C}$ for about 24 hours. Ethylaluminum dichloride $\left.(\mathrm{EtAlCl})_{2}\right)$ was distilled in vacuo before use. Polymerization was carried out according to the following procedure. A dry glass ampoule was evacuated and flushed with pure nitrogen gas and then dipped in a $-78^{\circ} \mathrm{C}$ bath. Acrylonitrile, liquid butadiene cooled at $-78^{\circ} \mathrm{C}, \mathrm{VOCl}_{3}$ dissolved in $n$-hexane and $\mathrm{EtAlCl}_{2}$ dissolved in $n$-hexane were added in this order by means of a hypodermic syringe. The ampoule was fused, sealed and dipped in an ice-water bath. After a given time in the dark, a small amount of methanol containing $N$-phenyl- $\beta$-naphthylamine was added to the reaction mixture which was subsequently poured into a large excess of hot water. The elastic polymer was coagulated, dried in vacuo and weighed. Conversion $(x \times 100)$ was estimated according to the following equation

$$
x=\frac{\text { Yield }(\mathrm{g})}{\text { the Monomer }(\mathrm{BD} / \mathrm{AN}=1 / 1)(\mathrm{g})}
$$

where the monomer is regarded as being composed of an equimolar amount of butadiene and acrylonitrile.

Polymerization was for the most part carried out at $0^{\circ} \mathrm{C}$ in the dark under the following conditions: the initial concentration of acrylonitrile being $11.46 \mathrm{~mol} / \mathrm{l}$, that of butadiene $2.01 \mathrm{~mol} / l$, that of $\mathrm{EtAlCl}_{2} 79.9 \times 10^{-3} \mathrm{~mol} / l$ and that of $\mathrm{VOCl}_{3} 7.99 \times 10^{-3} \mathrm{~mol} / l$. Under the polymeriza- tion conditions examined in these experiments, no gel-polymer formation was observed.

Intrinsic viscosities of the polymers were determined in $\mathrm{N}, \mathrm{N}$-dimethylformamide at $30^{\circ} \pm$ $0.05^{\circ} \mathrm{C}$ with an Ubbelohde type viscometer using the dilution method. The number-average molecular weight of copolymer $\left(\bar{M}_{n}\right)$ was calculated by using the following equation ${ }^{8}$

$$
[\eta]=3.91 \times 10^{-3} \bar{M}_{n}^{0.521}
$$

where $[\eta]$ is the intrinsic viscosity in $\mathrm{d} l / \mathrm{g}$.

\section{RESULTS AND DISCUSSION}

The effects of the concentrations of $\mathrm{EtAlCl}_{2}$, $\mathrm{VOCl}_{3}$ and of that of the sum of $\mathrm{EtAlCl}_{2}$ and $\mathrm{VOCl}_{3}$ concentrations on the copolymerization were investigated from the experimental relation of the polymerization time $v s$. the yield, intrinsic viscosity and composition of the copolymer. Similarly, the effects of the ratios of $\mathrm{VOCl}_{3}$ to EtAlCl ${ }_{2}$ at a given constant total concentration and those of the polymerization temperature were also observed. All the reaction mixtures in the experiments were homogeneous solutions. They became viscous at high conversion. The original data observed are summarized in Tables I, II, III, IV, and V.

As previously reported, ${ }^{4-6}$ the catalyst system is composed of alkylaluminum halide and a small amount of vanadium compound. It resembles the Ziegler catalyst in appearance but is quite different from it in its catalyst nature, because alkylaluminum halide itself is capable of inducing polymerization and the vanadium compound seems to act as a regenerator of the aluminum catalyst (see Figure 13). Basic additives such as tetrahydrofuran, pyridine, and triphenylphosphine completely disrupt polymerization, and weak bases such as dibutyl ether and thiophene diminish both the rate and degree of polymerization. ${ }^{6}$ Normal radical initiators and inhibitors are rather ineffective in regard to polymerization in this case, where the $\mathrm{Et}_{\mathrm{AlCl}}-\mathrm{VOCl}_{3}$ system at $0^{\circ} \mathrm{C}$ is used. Diphenylpicrylhydrazyl has little effect on the retardation, and sulfur and anthraquinone do not affect the polymerization. Benzoyl peroxide has no significant effect on the initiation. ${ }^{9}$ 
Alternating Copolymerization

Table I. Effects of the concentration of $\mathrm{VOCl}_{3}{ }^{\mathrm{a}}$

\begin{tabular}{|c|c|c|c|c|c|c|}
\hline No. & $\begin{array}{c}\text { Polymerization } \\
\text { time } \\
\text { (hr:min) }\end{array}$ & $\begin{array}{l}\text { Yield } \\
(\mathrm{g})\end{array}$ & $\begin{array}{c}\text { Conversion } \\
(\%)\end{array}$ & $\left(\begin{array}{l}{[\eta]} \\
(\mathrm{d} l / \mathrm{g})\end{array}\right.$ & $\begin{array}{l}\text { [AN] in } \\
\text { polymer } \\
(\mathrm{mol} \%)\end{array}$ & $\underset{(\mathrm{mol} / l)}{\left[\mathrm{VOCl}_{3}\right]}$ \\
\hline 1I-1 & $1: 00$ & 0.136 & 2.4 & $0.38_{0}$ & 45.0 & \multirow{5}{*}{$2.50 \times 10^{-5}$} \\
\hline 2 & $3: 00$ & 0.311 & 5.5 & 0.549 & $\longrightarrow$ & \\
\hline 3 & $6: 00$ & 0.563 & 9.9 & $0.61_{2}$ & 48.9 & \\
\hline 4 & 9.05 & 0.750 & 13.2 & $0.66_{9}$ & - & \\
\hline 5 & $18: 00$ & 1.275 & 22.4 & $0.72_{1}$ & 50.6 & \\
\hline $1 \mathrm{~J}-1$ & 1:00 & 0.146 & 2.6 & $0.38_{8}$ & 46.1 & \multirow{5}{*}{$4.00 \times 10^{-2}$} \\
\hline 2 & $2: 05$ & 0.279 & 4.9 & $0.47_{0}$ & - & \\
\hline 3 & $5: 00$ & 0.586 & 10.3 & $0.54_{6}$ & 50.8 & \\
\hline 4 & $9: 00$ & 0.942 & 16.5 & $0.57_{3}$ & - & \\
\hline 5 & $18: 50$ & 1.612 & 28.3 & $0.59_{8}$ & 51.5 & \\
\hline $1 \mathrm{M}-1$ & 1:00 & 0.189 & 3.3 & $0.36_{0}$ & 48.9 & \multirow{6}{*}{$7.99 \times 10^{-8}$} \\
\hline 2 & $2: 05$ & 0.375 & 6.6 & $0.42_{4}$ & - & \\
\hline 3 & $4: 05$ & 0.667 & 11.7 & $0.45_{1}$ & 49.7 & \\
\hline 4 & $8: 00$ & 1.161 & 20.4 & $0.47_{8}$ & - & \\
\hline 5 & $20: 00$ & 2.175 & 38.2 & $0.53_{5}$ & 51.4 & \\
\hline 6 & $20: 15$ & 2.366 & 41.5 & $0.54_{7}$ & 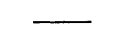 & \\
\hline $1 K-1$ & $1: 00$ & 0.320 & 5.6 & $0.33_{7}$ & 47.8 & \multirow{5}{*}{$20.0 \times 10^{-5}$} \\
\hline 2 & $2: 00$ & 0.564 & 9.9 & $0.40_{7}$ & $\longrightarrow$ & \\
\hline 3 & $4: 00$ & 1.040 & 18.3 & $0.44_{0}$ & 50.6 & \\
\hline 4 & 9:05 & 1.961 & 34.4 & $0.45_{8}$ & & \\
\hline 5 & $18: 10$ & 3.311 & 58.1 & $0.50_{1}$ & 51.4 & \\
\hline
\end{tabular}

a Polymerization conditions; [BD], $2.01 \mathrm{~mol} / l,[\mathrm{AN}], 11.46 \mathrm{~mol} / l,\left[\mathrm{EtAlCl}_{2}\right], 79.9 \times 10^{-3} \mathrm{~mol} / l$, Total volume, $26.5 \mathrm{ml}$, Theoretical yield, $5.70 \mathrm{~g}$.

Table II. Effects of the concentration of $\mathrm{EtAlCl}_{2}{ }^{\mathrm{a}}$

\begin{tabular}{|c|c|c|c|c|c|c|}
\hline No. & $\begin{array}{c}\text { Polymerization } \\
\text { time } \\
\text { (hr:min) }\end{array}$ & $\begin{array}{l}\text { Yield } \\
(\mathrm{g})\end{array}$ & $\begin{array}{c}\text { Conversion } \\
(\%)\end{array}$ & $\begin{array}{c}{[\eta]} \\
(\mathrm{d} l / \mathrm{g})\end{array}$ & $\begin{array}{l}\text { [AN] in } \\
\text { polymer } \\
(\mathrm{mol} \%)\end{array}$ & $\begin{array}{l}{\left[\mathrm{EtAlCl}{ }_{2}\right]} \\
(\mathrm{mol} / l)\end{array}$ \\
\hline $1 \mathrm{~S}-1$ & $1: 30$ & 0.064 & 1.1 & $0.17_{8}$ & - & \\
\hline 2 & $3: 00$ & 0.090 & 1.6 & $0.37_{3}$ & - & \\
\hline 3 & $9: 00$ & 0.140 & 2.5 & $0.80_{5}$ & 46.9 & $20.0 \times 10^{-3}$ \\
\hline 4 & $15: 00$ & 0.208 & 3.7 & $0.83_{8}$ & - & \\
\hline 5 & $24: 00$ & 0.240 & 4.2 & $1.11_{4}$ & 47.5 & \\
\hline $1 \mathrm{~T}-1$ & $1: 00$ & 0.071 & 1.3 & $0.25_{2}$ & - & \\
\hline 2 & $2: 00$ & 0.128 & 2.3 & 0.288 & - & \\
\hline 3 & $4: 00$ & 0.202 & 3.6 & $0.41_{5}$ & $\longrightarrow$ & $40.0 \times 10^{-3}$ \\
\hline 4 & $8: 00$ & 0.357 & 6.3 & $0.53_{7}$ & - & \\
\hline 5 & $21: 00$ & 0.876 & 15.4 & $0.62_{4}$ & - & \\
\hline $1 \mathrm{U}-1$ & $0: 45$ & 0.106 & 1.9 & 0.229 & - & \\
\hline 2 & $1: 45$ & 0.208 & 3.7 & $0.35_{6}$ & 48.5 & \\
\hline 3 & $4: 00$ & 0.540 & 9.5 & 0.449 & $\longrightarrow$ & $79.9 \times 10^{-3}$ \\
\hline 4 & $8: 00$ & 0.912 & 16.0 & $0.46_{1}$ & 49.9 & \\
\hline 5 & $19: 30$ & 1.909 & 33.5 & $0.55_{2}$ & 51.1 & \\
\hline $1 \mathrm{~V}-1$ & $0: 15$ & 0.137 & 2.4 & $0.24_{8}$ & - & \\
\hline 2 & $0: 30$ & 0.208 & 3.7 & $0.30_{4}$ & - & \\
\hline 3 & $1: 00$ & 0.367 & 6.4 & $0.38_{2}$ & 49.5 & \\
\hline 4 & $2: 00$ & 0.753 & 13.2 & $0.41_{0}$ & 48.0 & $\times 10^{-3}$ \\
\hline 5 & $5: 00$ & 1.516 & 26.6 & $0.49_{2}$ & 49.5 & \\
\hline 6 & $10: 00$ & 2.757 & 48.4 & $0.58_{5}$ & 49.5 & \\
\hline
\end{tabular}

a Polymerization conditions; [BD], $2.01 \mathrm{~mol} / l,[\mathrm{AN}], 11.46 \mathrm{~mol} / l,\left[\mathrm{VOCl}_{3}\right], 7.99 \times 10^{-3} \mathrm{~mol} / l$, Total volume, $26.5 \mathrm{ml}$, Theoretical yield, $5.70 \mathrm{~g}$. 
J. Furukawa, E. Kobayashi, and Y. Iseda

Table III. Effects of polymerization temperature ${ }^{a}$

\begin{tabular}{|c|c|c|c|c|c|c|}
\hline No. & $\begin{array}{c}\text { Polymerization } \\
\text { time } \\
\text { (hr:min) }\end{array}$ & $\begin{array}{l}\text { Yield } \\
(\mathrm{g})\end{array}$ & $\begin{array}{c}\text { Conversion } \\
(\%)\end{array}$ & $\begin{array}{c}{[\eta]} \\
(\mathrm{d} l / \mathrm{g})\end{array}$ & $\begin{array}{l}{[\mathrm{AN}] \text { in }} \\
\text { polymer } \\
(\mathrm{mol} \%)\end{array}$ & $\begin{array}{l}\text { Polymerization } \\
\text { temp. } \\
\left({ }^{\circ} \mathrm{C}\right)\end{array}$ \\
\hline $3 \mathrm{~K}^{\prime}-1$ & $1: 40$ & 0.097 & 1.7 & $0.30_{7}$ & - & \multirow{5}{*}{0.0} \\
\hline 2 & $4: 02$ & 0.170 & 3.0 & $0.47_{8}$ & 46.0 & \\
\hline 3 & 8:00 & 0.313 & 5.5 & $0.54_{2}$ & - & \\
\hline 4 & $12: 15$ & 0.499 & 8.8 & $0.55_{8}$ & 47.8 & \\
\hline 5 & $25: 30$ & 0.978 & 17.2 & $0.63_{8}$ & 49.9 & \\
\hline $3 \mathrm{~L}-1$ & $0: 30$ & 0.077 & 1.4 & $0.37_{3}$ & - & \multirow{5}{*}{15.0} \\
\hline 2 & $1: 00$ & 0.157 & 2.8 & $0.43_{2}$ & 45.0 & \\
\hline 3 & $2: 15$ & 0.305 & 5.4 & $0.51_{4}$ & - & \\
\hline 4 & $5: 38$ & 0.804 & 14.1 & $0.53_{3}$ & 48.8 & \\
\hline 5 & $10: 05$ & 1.204 & 21.1 & $0.57_{9}$ & 50.8 & \\
\hline $3 M-1$ & $0: 25$ & 0.215 & 3.8 & $0.40_{8}$ & 47.4 & \multirow{5}{*}{30.0} \\
\hline 2 & $1: 00$ & 0.485 & 8.5 & $0.45_{2}$ & - & \\
\hline 3 & $2: 10$ & 0.921 & 16.2 & $0.46_{8}$ & 51.0 & \\
\hline 4 & $4: 10$ & 1.523 & 26.7 & $0.51_{8}$ & - & \\
\hline 5 & $8: 00$ & 2.090 & 36.7 & $0.62_{9}$ & 53.1 & \\
\hline
\end{tabular}

a Polymerization conditions; [BD], $2.09 \mathrm{~mol} / l,[\mathrm{AN}], 11.92 \mathrm{~mol} / l,\left[\mathrm{EtAlCl}_{2}\right], 41.6 \times 10^{-3} \mathrm{~mol} / l, \quad\left[\mathrm{VOCl}_{3}\right.$ ], $4.08 \times 10^{-3} \mathrm{~mol} / l$, Total volume, $25.5 \mathrm{ml}$, Theoretical yield, $5.70 \mathrm{~g}$.

The characteristic feature of this sort of polymerization is a step-growth type as is shown in Figure 1. In this case, the degree of polymerization is calculated by taking the polymer composition to be $1: 1$. At extremely low conversion the polymer composition deviates somewhat from $1: 1$, but this is not sufficiently significant for the degree of polymerization to require correction. The degree of polymerization increases with conversion but at high conversion the increase is diminished.

Indeed, the number of polymer molecules is

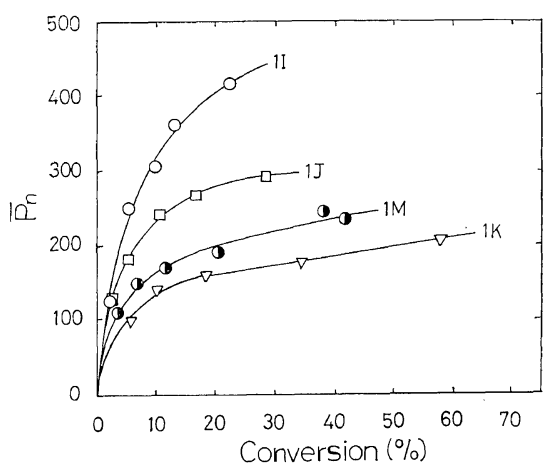

Figure 1. The plots of the degree of polymerization against conversion, $\left[\mathrm{VOCl}_{3}\right]$ being $2.50,4.00,7.99$, and $20.0 \mathrm{mmol} / l$ for experimental numbers $1 \mathrm{I}, 1 \mathrm{~J}$, $1 \mathrm{M}$, and $1 \mathrm{~K}$, respectively; data from Table $\mathrm{I}$. found to increase with polymer yield. In Figure 2 the ratio of the polymer yield $(Y)$ to the degree of polymerization $\left(\bar{P}_{n}\right)$ is plotted against the polymer yield.

The figure indicates that there is no induction period and linear relations are observed from the initial stage up to about $40 \%$ conversion. This means that polymerization involves rapid initiation and chain transfer to a considerable degree. The total concentration of active species generated can be estimated from the ordinate at zero yield and the relative rate of transfer from the slope

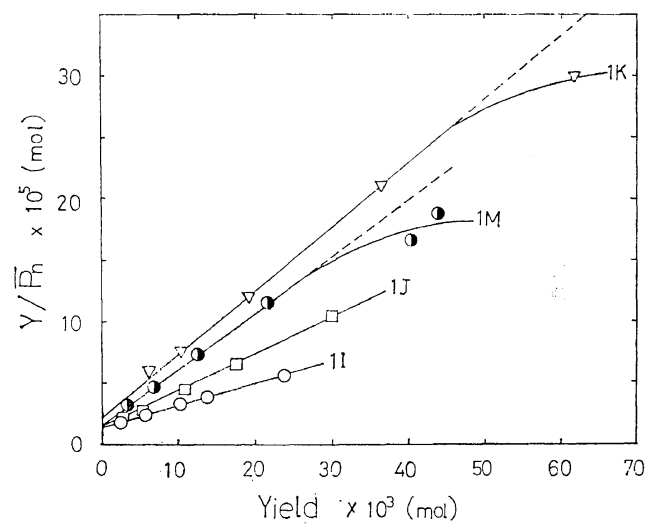

Figure 2. The plots of the ratio of polymer yield to the degree of polymerization against polymer yield; data from Table I. 


\section{Alternating Copolymerization}

Table IV-a. Effects of the total concentration of $\mathrm{EtAlCl}_{2}$ and $\mathrm{VOCl}_{3}$ (Polymerization conditions)

\begin{tabular}{ccccccc}
\hline No. & $\begin{array}{c}{[\mathrm{BD}]} \\
(\mathrm{mol} / l)\end{array}$ & $\begin{array}{c}{[\mathrm{AN}]} \\
(\mathrm{mol} / l)\end{array}$ & $\begin{array}{c}{[\mathrm{EtAlCl}]} \\
(\mathrm{mol} / l)\end{array}$ & $\begin{array}{c}{\left[\mathrm{VOCl}_{3}\right]} \\
(\mathrm{mol} / l)\end{array}$ & $\begin{array}{c}\text { Total } \\
\text { volume } \\
(\mathrm{m} l)\end{array}$ & $\begin{array}{c}\text { Theoretical } \\
\text { yield } \\
(\mathrm{g})\end{array}$ \\
\hline $1 \mathrm{O}$ & 2.01 & 11.46 & $40.0 \times 10^{-3}$ & $4.00 \times 10^{-3}$ & 26.5 & 5.70 \\
$1 \mathrm{P}$ & 2.01 & 11.46 & $79.9 \times 10^{-3}$ & $7.99 \times 10^{-3}$ & 26.5 & 5.70 \\
1Q & 2.01 & 11.46 & $160 \times 10^{-3}$ & $16.0 \times 10^{-3}$ & 26.5 & 5.70 \\
1R & 2.01 & 11.46 & $320 \times 10^{-3}$ & $32.0 \times 10^{-3}$ & 26.5 & 5.70 \\
\hline
\end{tabular}

Table IV-b. Effects of the total concentration of EtAlCl 2 and $\mathrm{VOCl}_{3}$ (Results observed)

\begin{tabular}{|c|c|c|c|c|c|}
\hline No. & $\begin{array}{c}\text { Polymerization } \\
\text { time } \\
\text { (hr:min) }\end{array}$ & $\begin{array}{c}\text { Yield } \\
(\mathrm{g})\end{array}$ & $\begin{array}{c}\text { Conversion } \\
(\%)\end{array}$ & $\begin{array}{c}[\eta]] \\
(\mathrm{d} l / \mathrm{g})\end{array}$ & $\begin{array}{l}{[\mathrm{AN}] \text { in }} \\
\text { polymer } \\
(\mathrm{mol} \%)\end{array}$ \\
\hline $10-1$ & $1: 08$ & 0.093 & 1.6 & $0.34_{8}$ & - \\
\hline 2 & 2:00 & 0.141 & 2.5 & $0.44_{5}$ & 48.1 \\
\hline 3 & $4: 00$ & 0.227 & 4.0 & $0.51_{7}$ & - \\
\hline 4 & $8: 00$ & 0.431 & 7.6 & $0.57_{0}$ & 50.0 \\
\hline 5 & 20:00 & 0.815 & 14.3 & $0.60_{5}$ & 50.0 \\
\hline $1 \mathrm{P}-1$ & $0: 45$ & 0.110 & 1.9 & $0.30_{8}$ & - \\
\hline 2 & $1: 45$ & 0.277 & 4.9 & $0.38_{0}$ & - \\
\hline 3 & $4: 10$ & 0.595 & 10.4 & $0.44_{0}$ & - \\
\hline 4 & 8:00 & 1.083 & 19.0 & $0.46_{5}$ & $\longrightarrow$ \\
\hline 5 & $18: 10$ & 2.008 & 35.2 & $0.52_{1}$ & - \\
\hline 1Q-1 & $0: 30$ & 0.242 & 4.2 & $0.28_{5}$ & 49.7 \\
\hline 2 & $1: 10$ & 0.617 & 10.8 & $0.33_{0}$ & - \\
\hline 3 & $2: 10$ & 1.129 & 19.8 & $0.34_{1}$ & 49.5 \\
\hline 4 & $6: 00$ & 2.557 & 44.9 & $0.42_{8}$ & - \\
\hline 5 & $15: 00$ & 4.450 & 78.1 & $0.51_{1}$ & 52.9 \\
\hline $1 \mathrm{R}-1$ & $0: 20$ & 0.903 & 15.8 & $0.25_{6}$ & 49.2 \\
\hline 2 & $0: 40$ & 1.501 & 26.3 & $0.28_{8}$ & - \\
\hline 3 & $1: 00$ & 2.214 & 38.8 & $0.31_{8}$ & 50.4 \\
\hline 4 & $3: 00$ & 4.809 & 84.4 & $0.40_{5}$ & 52.2 \\
\hline 5 & $9: 00$ & 6.174 & 108.3 & $0.38_{7}$ & 55.4 \\
\hline
\end{tabular}

of the straight line. Similar results can also be obtained from the reciprocal plots of the degree of polymerization against polymer yield. In fact the relative rate of transfer was evaluated from the former method and the concentration of active species from the latter method from the slopes of the straight lines. Therefore, from Figure 2

$$
Y \mid \bar{P}_{n}=a+b Y
$$

On the other hand, $Y \mid \bar{P}_{n}$ can be usually expressed as follows

$$
Y / \bar{P}_{n}=\int R_{i} d t-1 / 2 \int R_{t}{ }^{\prime} d t+\int R_{t r} d t
$$

where $R_{i}, R_{t}^{\prime}$, and $R_{t r}$ are rates of initiation, termination through recombination, and transfer, respectively. Comparing Eq. 1 with Eq. 2 it follows that

$$
\begin{gathered}
\int R_{i} d t-1 / 2 \int R_{t}{ }^{\prime} d t=a \\
\int R_{t r} d t / \int R_{p} d t=b
\end{gathered}
$$

Constants $a$ and $b$ were found to be affected by the catalyst and cocatalyst as shown in Table VI.

On the other hand, polymerization proceeds apparently in the first order with respect to the monomer concentration from the initial stage up 


\section{J. Furukawa, E. Kobayashi, and Y. ISEDA}

Table V-a. Effects of the ratio of $\left[\mathrm{VOCl}_{3}\right]$ to $\left[\mathrm{Et} \mathrm{AlCl}_{2}\right]$ (Polymerization conditions)

\begin{tabular}{lccccccc}
\hline No. & $\begin{array}{c}{[\mathrm{BD}]} \\
(\mathrm{mol} / l)\end{array}$ & $\begin{array}{c}{[\mathrm{AN}]} \\
(\mathrm{mol} / l)\end{array}$ & $\begin{array}{c}{[\mathrm{EtAlCl}]} \\
(\mathrm{mol} / l)\end{array}$ & $\begin{array}{c}{\left[\mathrm{VOCl}_{3}\right]} \\
(\mathrm{mol} / l)\end{array}$ & $\begin{array}{c}\text { V/Al } \\
(\mathrm{molar} \text { ratio) }\end{array}$ & $\begin{array}{c}\text { Total } \\
\text { volume } \\
(\mathrm{m} l)\end{array}$ & $\begin{array}{c}\text { Theoretical } \\
\text { yield } \\
(\mathrm{g})\end{array}$ \\
\hline 3T & 1.99 & 11.38 & $82.1 \times 10^{-3}$ & $1.6 \times 10^{-3}$ & $1 / 50$ & 26.7 & 5.70 \\
3U & 1.99 & 11.38 & $80.9 \times 10^{-3}$ & $2.7 \times 10^{-3}$ & $1 / 30$ & 26.7 & 5.70 \\
3V & 1.99 & 11.38 & $79.4 \times 10^{-3}$ & $4.2 \times 10^{-3}$ & $1 / 19$ & 26.7 & 5.70 \\
3W & 1.99 & 11.38 & $77.9 \times 10^{-3}$ & $5.7 \times 10^{-3}$ & $1 / 14$ & 26.7 & 5.70 \\
3X & 1.99 & 11.38 & $74.2 \times 10^{-3}$ & $9.4 \times 10^{-3}$ & $1 / 8$ & 26.7 & 5.70 \\
3Y & 1.99 & 11.38 & $66.7 \times 10^{-3}$ & $16.9 \times 10^{-3}$ & $1 / 4$ & 26.7 & 5.70 \\
\hline
\end{tabular}

Table $\mathbf{V}-\mathbf{b}$. Effects of the ratio of $\left[\mathrm{VOCl}_{3}\right]$ to $\left[\mathrm{EtAlCl} \mathrm{Al}_{2}\right]$ (Results observed)

\begin{tabular}{|c|c|c|c|c|c|}
\hline No. & $\begin{array}{c}\text { Polymerization } \\
\text { time } \\
\text { (hr:min) }\end{array}$ & $\begin{array}{l}\text { Yield } \\
(\mathrm{g})\end{array}$ & $\begin{array}{c}\text { Conversion } \\
(\%)\end{array}$ & $\begin{array}{l}{[\eta]} \\
(\mathrm{d} l / \mathrm{g})\end{array}$ & $\begin{array}{l}{[\mathrm{AN}] \text { in }} \\
\text { polymer } \\
(\mathrm{mol} \%)\end{array}$ \\
\hline $3 \mathrm{~T}-1$ & $1: 30$ & 0.160 & 2.8 & $0.47_{2}$ & - \\
\hline 2 & $3: 25$ & 0.313 & 5.5 & $0.57_{5}$ & 45.5 \\
\hline 3 & $6: 05$ & 0.487 & 8.6 & $0.64_{2}$ & - \\
\hline 4 & $9: 05$ & 0.615 & 10.8 & $0.66_{2}$ & 48.1 \\
\hline 5 & $20: 00$ & 1.140 & 20.0 & $0.76_{8}$ & 48.7 \\
\hline $3 \mathrm{U}-1$ & $1: 10$ & 0.157 & 2.8 & $0.35_{7}$ & - \\
\hline 2 & $2: 30$ & 0.305 & 5.4 & $0.50_{4}$ & 48.0 \\
\hline 3 & $5: 00$ & 0.531 & 9.3 & $0.53_{8}$ & 48.3 \\
\hline 4 & $10: 00$ & 0.923 & 16.2 & $0.58_{2}$ & - \\
\hline 5 & $20: 00$ & 1.551 & 27.2 & $0.68_{6}$ & 49.2 \\
\hline $3 \mathrm{~V}-1$ & $1: 20$ & 0.188 & 3.3 & $0.38_{0}$ & 47.5 \\
\hline 2 & $2: 00$ & 0.287 & 5.0 & $0.41_{2}$ & - \\
\hline 3 & $4: 20$ & 0.549 & 9.6 & $0.46_{2}$ & 47.9 \\
\hline 4 & $8: 00$ & 0.896 & 15.7 & $0.49_{0}$ & - \\
\hline 5 & $18: 00$ & 1.582 & 27.8 & $0.57_{6}$ & 50.0 \\
\hline $3 W-1$ & $1: 00$ & 0.183 & 3.2 & 0.290 & 43.3 \\
\hline 2 & $2: 00$ & 0.318 & 5.6 & $0.38_{4}$ & - \\
\hline 3 & $4: 05$ & 0.557 & 9.8 & $0.41_{7}$ & 49.3 \\
\hline 4 & $8: 00$ & 0.994 & 17.4 & $0.42_{7}$ & - \\
\hline 5 & $18: 02$ & 1.712 & 30.0 & $0.50_{7}$ & 49.8 \\
\hline $3 X-1$ & $0: 50$ & 0.163 & 2.9 & $0.27_{3}$ & - \\
\hline 2 & $1: 45$ & 0.320 & 5.6 & $0.33_{6}$ & 48.6 \\
\hline 3 & $5: 45$ & 0.917 & 16.1 & $0.41_{4}$ & 50.6 \\
\hline 4 & $9: 00$ & 1.302 & 22.8 & $0.41_{6}$ & - \\
\hline 5 & $17: 50$ & 2.010 & 35.3 & $0.45_{2}$ & 49.8 \\
\hline $3 Y-1$ & $0: 50$ & 0.194 & 3.4 & 0.24 .2 & - \\
\hline 2 & $2: 00$ & 0.453 & 8.0 & $0.32_{2}$ & 45.9 \\
\hline 3 & $4: 15$ & 0.832 & 14.6 & $0.37_{8}$ & $\longrightarrow$ \\
\hline 4 & $8: 00$ & 1.325 & 23.3 & $0.41_{1}$ & 49.3 \\
\hline 5 & $16: 20$ & 2.282 & 40.0 & $0.43_{5}$ & 50.0 \\
\hline
\end{tabular}




\section{Alternating Copolymerization}

Table VI. Effects of [EtAlCl$\left.{ }_{2}\right]$ and $\left[\mathrm{VOCl}_{3}\right]$ on $a$ and $b$

\begin{tabular}{lcccc}
\hline No. & $\begin{array}{c}{\left[\mathrm{VOCl}_{3}\right]} \\
(\mathrm{mmol} / l)\end{array}$ & $\begin{array}{c}{[\mathrm{EtAlCl}} \\
(\mathrm{mmol} / l)\end{array}$ & $\begin{array}{c}a \\
(\mathrm{mmol} / l)\end{array}$ & $b$ \\
\hline $1 \mathrm{~T}$ & 7.99 & 40.0 & 0.80 & $1.88 \times 10^{-3}$ \\
$1 \mathrm{U}$ & 7.99 & 79.9 & 1.25 & $3.40 \times 10^{-3}$ \\
$1 \mathrm{~V}$ & 7.99 & 160 & 1.46 & $3.72 \times 10^{-3}$ \\
$1 \mathrm{I}$ & 2.50 & 79.9 & 0.47 & $1.83 \times 10^{-3}$ \\
$1 \mathrm{~J}$ & 4.00 & 79.9 & 0.50 & $3.03 \times 10^{-3}$ \\
$1 \mathrm{M}$ & 7.99 & 79.9 & 0.64 & $4.63 \times 10^{-3}$ \\
$1 \mathrm{~K}$ & 20.0 & 79.9 & 1.28 & $5.17 \times 10^{-3}$ \\
\hline
\end{tabular}

Table VII. Effects of $[\mathrm{EtAlCl}]$ and $\left[\mathrm{VOCl}_{3}\right]$ on the initial rate of polymerization $\left(R_{p_{0}}\right)$ and the first-order rate constant $(k)$

\begin{tabular}{lcccc}
\hline No. & $\begin{array}{c}{\left[\mathrm{VOCl}_{3}\right]} \\
(\mathrm{mmol} / l)\end{array}$ & $\begin{array}{c}{[\mathrm{EtAlCl}} \\
(\mathrm{mmol} / l)\end{array}$ & $\begin{array}{c}R_{p_{0}} \\
(\mathrm{mmol} / l / \mathrm{hr})\end{array}$ & $\begin{array}{c}k \\
\left(\mathrm{hr}^{-1}\right)\end{array}$ \\
\hline $1 \mathrm{~S}$ & 7.99 & 20.0 & 10.4 & $0.25 \times 10^{-2}$ \\
$1 \mathrm{~T}$ & 7.99 & 40.0 & 38.5 & $0.78 \times 10^{-2}$ \\
$1 \mathrm{U}$ & 7.99 & 79.9 & 95.4 & $2.16 \times 10^{-2}$ \\
$1 \mathrm{~V}$ & 7.99 & 160 & 313 & $6.69 \times 10^{-2}$ \\
$1 \mathrm{I}$ & 2.50 & 79.9 & 78.3 & $1.66 \times 10^{-2}$ \\
$1 \mathrm{~J}$ & 4.00 & 79.9 & 98.5 & $2.03 \times 10^{-2}$ \\
$1 \mathrm{M}$ & 7.99 & 79.9 & 129 & $2.90 \times 10^{-2}$ \\
$1 \mathrm{~K}$ & 20.0 & 79.9 & 203 & $4.79 \times 10^{-2}$ \\
\hline
\end{tabular}

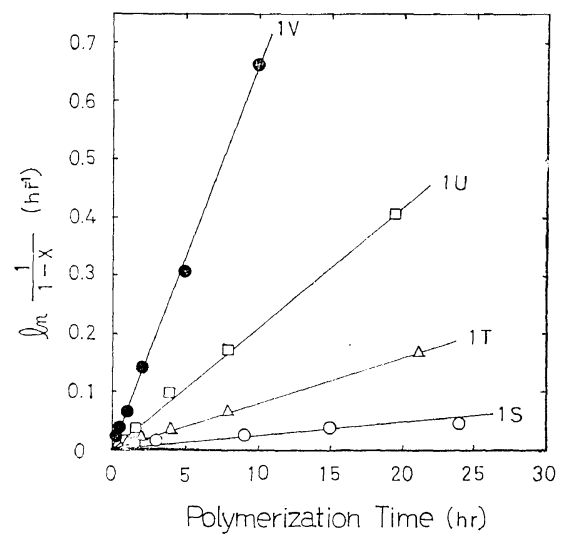

Figure 3. First order plots of copolymerization, [EtAlCl ${ }_{2}$ ] being 20.0, 40.0, 79.9, and $160 \mathrm{mmol} / \mathrm{l}$ for experimental numbers $1 \mathrm{~S}, 1 \mathrm{~T}, 1 \mathrm{U}$, and $1 \mathrm{~V}$, respectively; data from Table II.

to about $30 \%$ conversion as shown in Figure 3 and is expressed as follows

$$
\ln \frac{1}{1-x}=k t
$$

where $x$ is the conversion, $k$ the first-order rate constant and $t$ the reaction time. The initial rate $\left(R_{p_{0}}\right)$ and the apparent rate constant $(k)$ of polymerization thus obtained are listed as functions of the catalyst and cocatalyst in Table VII.

Activation energies of overall polymerization and transfer were evaluated from the Arrhenius plots in Figures 4 and 5 as follows.

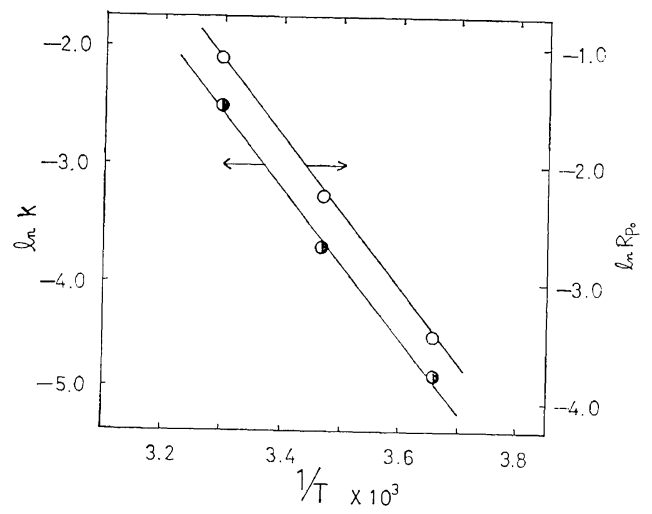

Figure 4. Arrhenius plots of overall polymerization, [BD], [AN], [EtAlCl 2$]$, and [ $\left[\mathrm{VOCl}_{3}\right]$ being 2.09, $11.92,41.6 \times 10^{-3}$, and $4.08 \times 10^{-3} \mathrm{~mol} / l$, respectively; data from Table III.

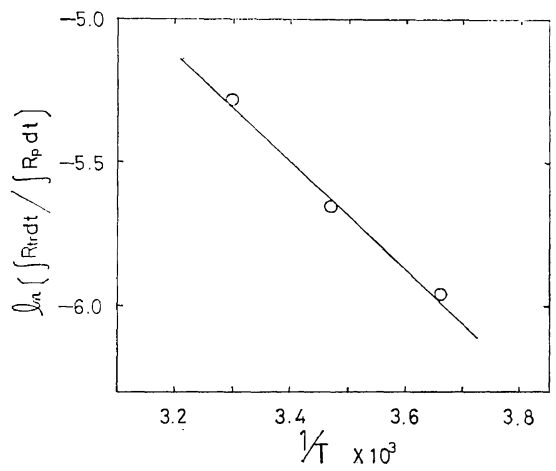

Figure 5. Arrhenius plots of transfer-propagation; data from Table III.

$$
E_{a}(\text { overall })=13.4 \mathrm{kcal} / \mathrm{mol}
$$

$E_{a}$ (transfer $)-E_{a}$ (propagation $)=3.7 \mathrm{kcal} / \mathrm{mol}$

The dependence of the concentrations of active species on temperature is found to be $1.1 \mathrm{kcal} / \mathrm{mol}$.

The effects of the catalyst and cocatalyst were investigated in detail. It was found that ethyl- 

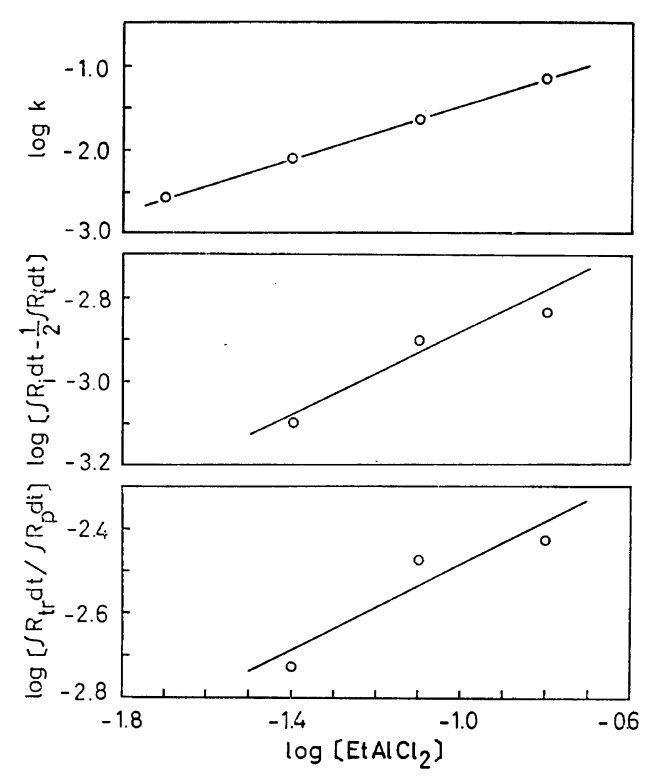

Figure 6. Effect of $\left[\mathrm{EtAlCl}_{2}\right]$ on the rate of polymerization, the concentration of active species, and $\int R_{t r} d t / \int R_{p} d t ;$ data from Table II.
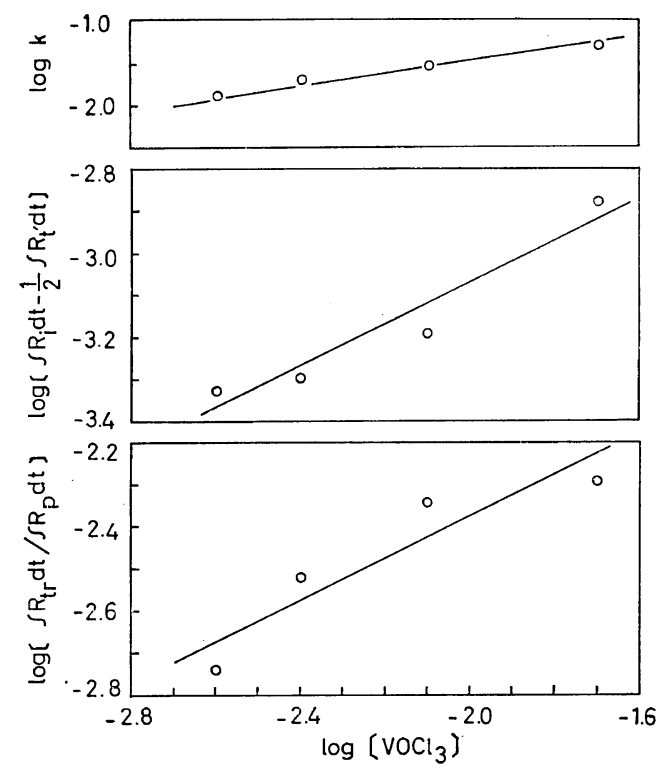

Figure 7. Effect of $\left[\mathrm{VOCl}_{3}\right]$ on the rate of polymerization, the concentration of active species, and $\int R_{t r} d t / \int R_{p} d t$; data from Table I. aluminum dichloride enhances the rate of polymerization, transfer, and the formation of active species. The cocatalyst was found to be effective not only for increasing catalyst activity but also for transfer reaction.

From Figures 6 and 7 the order is evaluated as follows although the data on the chain transfer are too rough to determine its order.

$$
\begin{aligned}
R_{p_{0}} & \propto\left[\mathrm{EtAlCl}_{2}\right]^{1.5}\left[\mathrm{VOCl}_{3}\right]^{0.5} \\
{\left[C^{*}\right] } & \propto\left[\mathrm{EtAlCl}_{2}\right]^{0.5}\left[\mathrm{VOCl}_{3}\right]^{0.5} \\
R_{t r} / R_{p} & \propto\left[\mathrm{EtAlCl}_{2}\right]^{0.5}\left[\mathrm{VOCl}_{3}\right]^{0.5}
\end{aligned}
$$

Where $\left[C^{*}\right]$ is the total concentration of the active species. As a result, $R_{t r}$ is tentatively expressed as

$$
R_{t r} \propto k_{t r}\left[\mathrm{EtAlCl}_{2}\right]^{2}\left[\mathrm{VOCl}_{3}\right]
$$

The Eqs. 8-11 are also well supported by the results obtained as shown in Tables IV and V. These kinetic data suggest that the polymerization proceeds in the manner of step-growth with rapid initiation and some chain transfer. The concentration of the active species at the initial stage seems to be proportional to the square root of the aluminum and vanadium component, respectively. This fact can be interpreted by assuming that the active species arises from the dissociation of the complex of both components, as follows.

$$
\begin{aligned}
& \mathrm{EtAlCl}_{2}+\mathrm{VOCl}_{3} \rightleftharpoons\left[\mathrm{EtA}^{\mathrm{AlCl}} \mathrm{H}_{3} \longleftarrow \stackrel{+}{\mathrm{V}} \mathrm{OCl}_{2}\right] \\
& \rightleftarrows \text { dissociated into radicals }
\end{aligned}
$$

Although the actual radical responsible for initiation has still to be studied further it seems likely to be a kind of redox system. In this case acrylonitrile appears to participate in the initiation, because no initiation takes place in its absence. Since the polymerization is a stepgrowth type, the rate of polymerization is expressed as a function of the concentration of radical produced and concentrations of monomers. From Eq. 8 the actual monomer is believed to be complexed with the aluminum compound and consequently, it follows that

$$
\begin{aligned}
R_{p} \propto & k_{p}\left\{k_{i}\left[\mathrm{EtAlCl}_{2}\right]^{0.5}\left[\mathrm{VOCl}_{3}\right]^{0.5}\right\} \\
& \times\left\{[\mathrm{Monomer}]\left[\mathrm{EtAlCl}_{2}\right]\right\}
\end{aligned}
$$

where [Monomer] implies the concentration of the $1: 1$ combination of both monomers. 


\section{Alternating Copolymerization}

Polymerization may take place between complexed radicals and complexed monomers. For instance:

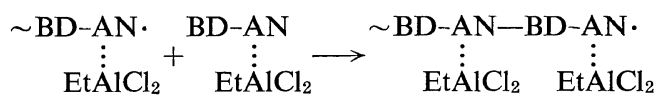

The vanadium compound seems to bring about the recycle of the aluminum compound complexed with the nitrile group in the polymer chain to the active species, because in the absence of the vanadium compound the yield of the copolymer hardly exceeds the amount of the aluminum compound used as shown in Figure 8.

The chain transfer reaction expressed by Eq. 11 is somewhat complicated but it is related to both the aluminum and vanadium compounds. The transfer reaction may to some extent coexist with the reaction between the polymer terminal complexed with aluminum compound and the vanadium compound, as, for example, in the following form.
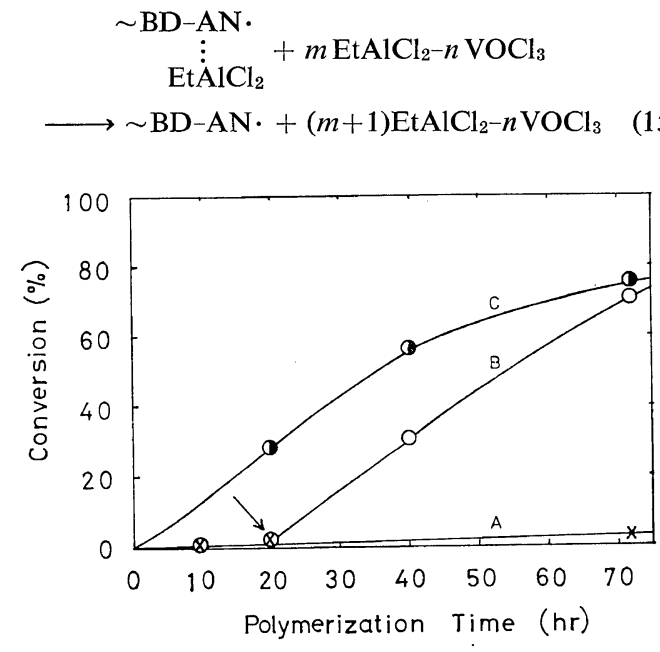

Figure 8. Effect of $\left[\mathrm{VOCl}_{3}\right]$ added after the long period of polymerization by $\left[\mathrm{EtAlCl}_{2}\right]$ alone; [BD], $39 \mathrm{mmol},[\mathrm{AN}], 276 \mathrm{mmol},\left[\mathrm{EtAlCl}_{2}\right], 1.55$ mmol.

A: Copolymerization by $\left[\mathrm{EtAlCl}_{2}\right]$ alone.

B: Copolymerization by $\left[\mathrm{EtAlCl}_{2}\right]$ alone for initial $20 \mathrm{hr}$ and later together with additional $\left[\mathrm{VOCl}_{3}\right]$ added at the point shown by an arrow; $\left[\mathrm{VOCl}_{3}\right]$ $0.16 \mathrm{mmol}$.

C: Copolymerization by the $\mathrm{EtAlCl}_{2}-\mathrm{VOCl}_{3}$ system.
Experimental data indicate that acrylonitrile appears to participate in the above catalysttransfer reaction, because the value of $R_{t r} / R_{p}$ is also proportional to $[\mathrm{AN}]^{2.3}{ }^{.}$From kinetic data $m$ is estimated to be 1.5 and $n$ to be 0.5 .

The most important feature of this type of polymerization may lie in the polymerization of the active monomer complexed with a catalyst. The degree of the alternating tendency in the copolymer chain was measured by $100 \mathrm{Mc}$ NMR analysis with the copolymers obtained in the above experiments and found to be about $95 \%{ }^{9}$ According to the IR analyses of the above copolymers, the butadiene unit was always linked in trans-1,4. The influence of the concentration of the monomer on the rate is complicated, and it will be discussed elsewhere.

We are grateful to Dr. T. Yoshimoto and $\mathrm{K}$. Tanaka for NMR measurements and to Miss K. Giga for experimental assistance.

\section{REFERENCES}

1. M. Hirooka, H. Yabuuchi, S. Morita, S. Kawasumi, and K. Nakaguchi, J. Polym. Sci., Part B, 5, 47 (1967).

2. M. Hirooka, H. Yabuuchi, J. Iseki, and Y. Nakai, ibid., Part A-1, 6, 1381 (1968).

3. N. G. Gaylord and A. Takahashi, paper presented at the Annual ACS Meeting, San Francisco, 1968.

4. J. Furukawa and Y. Iseda, J. Polym. Sci., Part B, 7, 47 (1969).

5. J. Furukawa, Y. Iseda, K. Haga, N. Kataoka, T. Yoshimoto, T. Imamura, Y. Shido, A. Miyagi, K. Tanaka, and K. Sakamoto, paper presented at the Annual Meeting of the Society of Polym. Sci. of Japan, Tokyo, 1968; J. Polym. Sci., Part B, 7, 561 (1969).

6. J. Furukawa, Y. Iseda, K. Haga, and N. Kataoka, paper presented at the Annual Meeting of the Chem. Soc. of Japan, Tokyo, 1969; J. Polym. Sci., Part A-1, in press.

7. M. Taniguchi, A. Kawasaki, and J. Furukawa, paper presented at the Annual Meeting of the Chem. Soc. of Japan, Tokyo, 1969; J. Polym. Sci., Part B, 7, 411 (1969).

8. T. Yoshimoto, et al., private communication.

9. J. Furukawa, Y. Iseda, and E. Kobayashi, paper presented at the Symposium on High Polymers, Tokyo, 1969. 